Mercuric Sulfide.-Mercuric sulfide was found to react readily with thionyl chloride at $150^{\circ}$ with the formation of long needle crystals of mercuric chloride. Reaction proceeds according to the equation

$$
\begin{gathered}
\mathrm{HgS}+2 \mathrm{SOCl}_{2}=\mathrm{CuCl}_{2}+\mathrm{SO}_{2}+\mathrm{S}_{2} \mathrm{Cl}_{2} . \\
\text { Summary. }
\end{gathered}
$$

Thionyl chloride reacts with sulfides according to the following equation, in which $M$ represents a divalent metal:

$$
\mathrm{MS}+{ }_{2} \mathrm{SOCl}_{2}=\mathrm{MCl}_{2}+\mathrm{SO}_{2}+\mathrm{S}_{2} \mathrm{Cl}_{2}
$$

With sulfides as with oxides and metals, thionyl chloride seems to show a selective action, reacting much more readily with some than with others.

Naw Brunswick, N. I.

\title{
THE DETERMINATION OF GASES DISSOLVED IN WATERS AND EFFLUENTS.
}

By A. A. Swanson and G. A. Hulett.

Received September 9, 1915.

The importance of a knowledge of gases dissolved in natural waters and effuents has lead to the development of many methods for their estimation. The results obtained by these different methods are, however, not always concordant or satisfactory. Our attention was directed to this subject by some special difficulties encountered in the determination of the oxygen in mine waters and we were led to examine this whole subject of the determination of gases dissolved in waters and solutions. The method we have devised for our special purpose makes use of a principle which does not appear to have been used in this kind of work heretofore, but it has solved our problem and seems to be generally applicable.

The methods which have been used for the determination of gases dissolved in waters fall into two groups: First, those methods which are based on chemical reaction involving the gas in solution in a reaction which may be followed "titrimetrically." In the second group the gases are first removed from the water and then determined gasometrically. The chemical methods work well in the determination of oxygen and carbon dioxide, while no success has been attained in determining other dissolved gases such as nitrogen, owing to a lack of suitable chemical reactions.

At the present time most attention is given to the determination of oxygen and carbon dioxide in natural waters and effuents. The methods in favor for the determination of dissolved oxygen are the Winkler ${ }^{1}$ and Levy ${ }^{2}$ methods. A modification of the Winkler method by Hale and Melia ${ }^{3}$ has made it possible, however, to determine the dissolved

1 Ber., 21, 2843 (I888); 22, I764 (1889).

'Ann. l'obseratorie Municipal de Montosouris, Paris, 1884.

"J. Ind. Eng. Chem, 5, 976 (1913). 
oxygen content of waters containing nitrites. In the second group of methods the gases are first removed from the water either by boiling or under reduced pressure and then their composition determined gasometrically. In this class belong the methods devised by Bunsen, ${ }^{1}$ Roscoe and Lunt, ${ }^{2}$ Birge and Juday ${ }^{3}$ and Tenax. ${ }^{4}$ The accuracy of some of these methods is not questioned, but their adaptability for general use is limited on account of the difficulty encountered in removing the last traces of the dissolved gases and in handling the gases removed, but it seemed to be the only method in certain cases, especially where the chemical method is limited by the nature of the water under investigation. For example, in attempting to determine the dissolved oxygen in mine waters the presence of both ferrous and ferric iron interferes with the usual procedure, or, in general, where the water contains an oxidizable or a reducible substance. But this objection does not apply to the methods of the second group, although here there is encountered the difficulty of removing the last traces of the gases, as has been stated, and the methods have therefore received little attention.

If, now, we take advantage of the law of distribution of a gas between the liquid and vapor phase it will not be necessary to remove any, or at least all, of the gases dissolved in the water or solution in order to determine its amount. The ideal method of determining the amount of the gases dissolved in a water or solution would be to prepare and know the composition of a gas phase which was in equilibrium with our water at a known temperature and we could then calculate with accuracy from known data each constituent in the water. While we have not found a method of doing this, it has been found possible to bring the water into a vacuum, and, by shaking, to bring the system to equilibrium; so that the dissolved gases are properly distributed between the gas and liquid phase, then on analyzing the gas phase and knowing the temperature and volumes we calculate with accuracy from known coefficients of distribution ${ }^{5}$ the amounts of gases in the two phases, and have the desired information. ${ }^{6}$

1 J. Chem. Soc., 55, 552 (1889).

${ }^{2}$ Ibid., 55, 552 (1889).

${ }^{3}$ Wisc. Survey Bull., 23 (I 911 ).

- Plöner Forschungs ber., I1, 82 (1904); Z. angew. Chem., 20, 1930 (1907); Süsswasser, 128 (Ig07).

- Landolt-Börnstein, Wied. Ann.; 44, 318 (1891).

- Dr. J. J. Johnson has just called our attention to an article by Fresenius and Gruenhut (Z. anal. Chem., 53, 265). These authors worked with a "Schuettlerohr" which was devised by $\mathrm{E}$. Reichardt for determining $\mathrm{CO}_{2}$ in mineral waters. The tube was partly filled with the water, closed and shaken, part of the $\mathrm{CO}_{2}$ entered the gas phase increased the pressure and was allowed to displace an equal volume of the water. The tube gave a rapid method for an approximate determination of the $\mathrm{CO}_{2}$ and was based on Henry's Law. 
The apparatus we used to carry out this idea consisted essentially of two glass reservoirs or bulbs, cylindrical in form and having a capacity of one liter, and one-half liter, respectively. They were fitted with "I 20 degree" three-way cocks at the upper ends and with straight cocks at the lower ends. A short-range thermometer was fastened to the inner

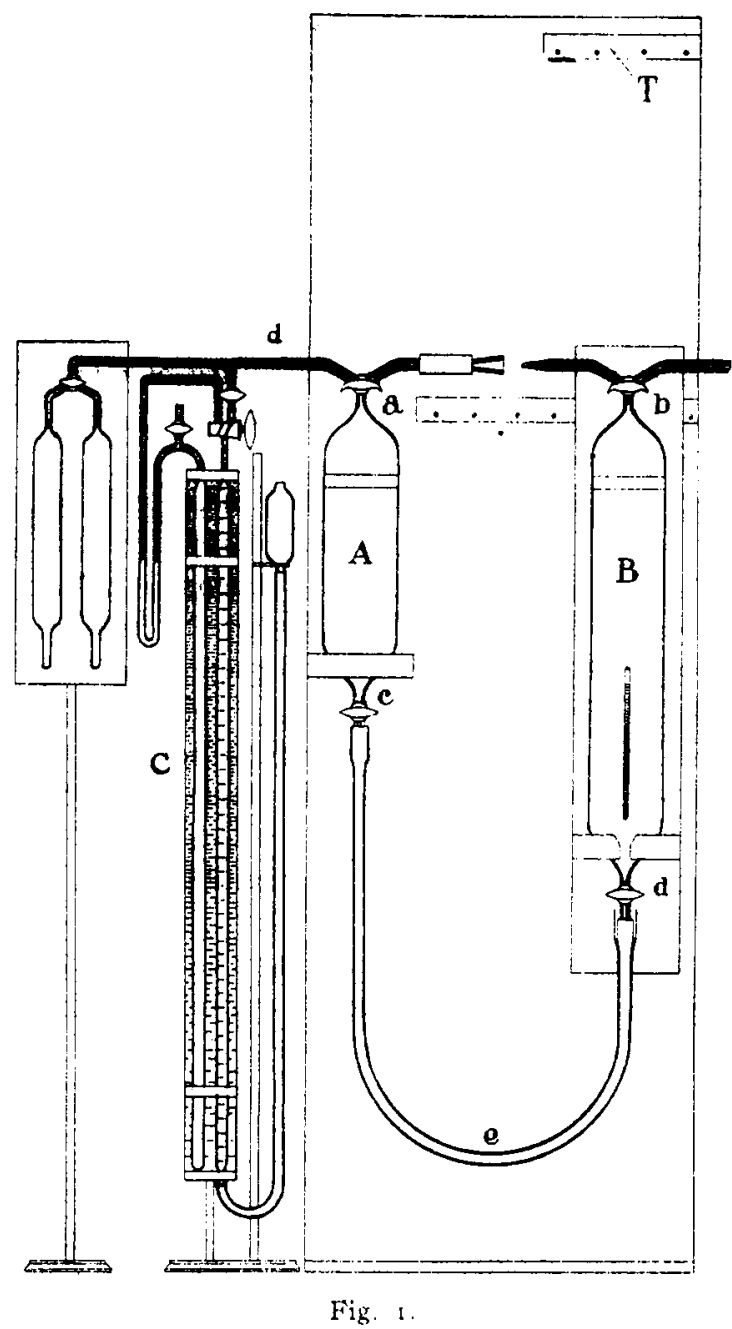
wall of the larger bulb so that it was possible to determine the temperature of the contents with certainty to $0.1^{\circ}$. 'Ihese bulbs were mounted on a frame as shown in Fig. I. 'The small bulb $A$ was permanently mounted while the larger bulb $B$ was so arranged that its carriage permitted of either a horizontal motion or of being raised vertically. Bulb $A$ was connected with a gas analysis apparatus of special design, (C) through a capillary tube, $d$. 'The two bulbs were made and mounted so that they could be joined at either end with each other at will.

'The water to be examined was drawn directly into bulb B and the cocks closed and there could then be no gain or loss of gases. In making a run bulb $B$, with the water under examination, was placed on its carriage and then joined to the lower end of bulb $A$, by means of suitable tubing and a mercury seal so that there was no air in the connections. On opening the lower cocks on bulbs $A$ and $B$, the mercury in $A$ flowed by gravity into the lower half of bulb $B$ and displaced an equal volume of water through the upper cock on bulb $B$. 'This water was reserved for any 
special tests. Cock $b$ was closed and the carriage with the bulb B raised to the position indicated at $T$ and the mercury allowed to flow back to bulb $A$, leaving the water in bulb $B$ in a vacuum; the lower cock on bulb $B$ was then closed and the bulb was removed from its support and shaken vigorously in order to bring the liquid and vapor phases into equilibrium. This operation required only a couple of minutes. The bulb $B$ was then replaced on the carriage and lowered to its original position and again connected with the lower end of bulb $A$. The carriage was then moved along the slide rail so that bulbs $A$ and $B$ were joined at the upper ends by means of a ground-glass joint. Cocks $a$ and $b$ were then made communicating and the line evacuated and mercury allowed to fill the line to the cock $b$. The system was then ready for a transfer of the gas from bulb $B$ to bulb $A$ without changing its pressure, an equal volume of mercury flowing from bulb $A$ to bulb $B$. Then the gas could safely be brought to any desired pressure over mercury, transferred to the gas buret, and analyzed in the usual way. It was not desirable to change the pressure of the gas while in contact with the water, as some of it would dissolve.

From the amount of the gases found, the volume and temperature when in equilibrium with the water in bulb $B$, and the known coefficients of distribution of the gases, the total amounts of the gases dissolved in the water was calculated. The following formulas were used to calculate the amount of dissolved gases in the waters examined:

$$
x=a\left(\frac{c(\mathrm{~T}+t)}{\mathrm{T}}+\mathrm{I}\right)
$$

Where $x=$ total number of cc. of the particular gas in the sample of water taken for analysis.

$a=$ number of $\mathrm{cc}$. of the desired gas actually removed.

$c=$ solubility coefficient of the gas at the temperature observed.

$t=$ temperature at which the gas and liquid phases were at equilibrium.

$T=$ absolute temperature of Centigrade scale.

In case the volume of the liquid and gas phases in the bulb were not equal the above equation was modified as follows:

$$
x=\frac{a}{b}\left(\frac{b c(T+t)}{T}+d\right)
$$

Where $b=$ volume of the liquid phase.

$d=$ volume of the vapor phase.

The accompanying Tables (I), (II), (III) of several series of determinations show the agreement we obtained by the use of this method in determining dissolved oxygen in various waters. The results thus found were calculated to standard conditions of temperature and pressure and are expressed in cubic centimeters of oxygen gas per liter of water 
as is customary. 'The method was first tested by running check determinations against the "Winkler" method." Distilled water thoroughly aerated was used for part of the tests, so there was nothing present which could be oxidized and nothing reduced save the oxygen, so the Winkler method should give correct values.

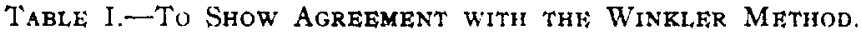

$\begin{array}{lccccc}\begin{array}{c}\text { Amt. of } \\ \text { oxygen } \\ \text { removed. }\end{array} & \begin{array}{c}\text { Equil. } \\ \text { temp. }\end{array} & \begin{array}{c}\text { Temp. of } \\ \text { anglysis } \\ \text { of gas. }\end{array} & \begin{array}{c}\text { Bar pres. } \\ \text { in mm. }\end{array} & \text { New meth. } & \begin{array}{c}\text { Winkler. } \\ \text { Cc. per liter. }\end{array} \\ 3.18 \mathrm{cc} . & 21.4^{\circ} & 21.3^{\circ} & 759.20 & 6.22 & 6.22 \\ 3.19 & 21.3 & 21.0 & 766.30 & 6.26 & 6.26 \\ 3.19 & 22.7 & 22.3 & 755.75 & 6.16 & 6.14 \\ 3.16 & 22.7 & 22.3 & 756.60 & 6.11 & 6.13 \\ 3.26 & 20.9 & 20.3 & 762.00 & 6.41 & 6.4 \mathrm{I} \\ 3.34 & 19.4 & 19.1 & 766.75 & 6.65 & 6.63 \\ 3.33 & 22.8 & 22.4 & 762.35 & 6.48 & 6.52 \\ 3.21 & 23.2 & 23.4 & 762.80 & 6.22 & 6.27 \\ 3.07 & 21.2 & 23.6 & 745.40 & 5.86 & 5.84 \\ 3.04 & 22.0 & 23.0 & 753.70 & 5.83 & 5.86 \\ 4.68 & 18.1 & 19.88 & 749.70 & 9.06 & 9.07\end{array}$

TABle II.--To SHOW The Reproducibility of OUR Method.

Distilled Water.

$\left.\begin{array}{lllll|l}3.00 \mathrm{cc} . & 23.0^{\circ} & 23.5^{\circ} & 749.90 & 5.708 \\ 3.02 & 23.4 & 24.0 & 748.85 & 5.730 \\ 3.01 & 22.9 & 23.8 & 749.25 & 5.717 \\ 3.00 & 22.6 & 23.5 & 749.25 & 5.703 \\ 2.98 & 22.5 & 22.4 & 750.15 & 5.705 \\ 3.00 & 23.0 & 23.0 & 750.15 & 5.724\end{array}\right\} \quad 5.707$

Table III.-To Show Agregment on Various Waters.

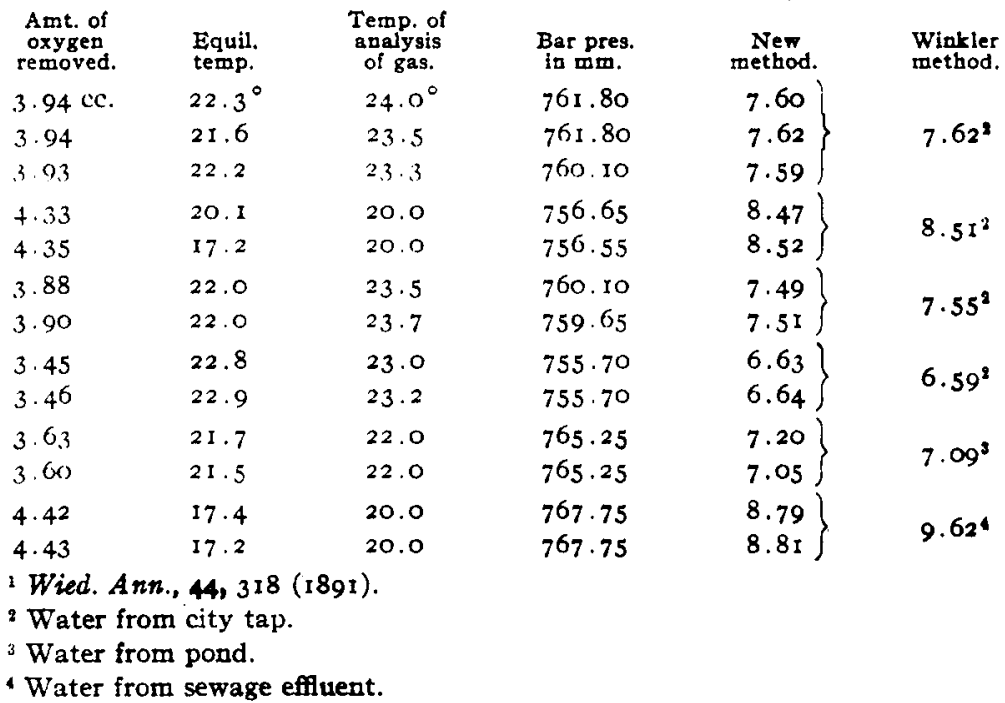




\section{Carbon Dioxide.}

Of the several methods which have been proposed for the determination of free carbon dioxide in water, the so-called Seyler's modification of the Pettenkofer ${ }^{1}$ method seems to be the one most easily adapted and the most reliable one. It has been fully described by Seyler in the Chemical News ${ }^{2}$ and recently has received exhaustive study of Ellms and Beneker, ${ }^{3}$ who reached the conclusion that it gives the most accurate results. It consists of titrating with sodium carbonate and phenolphthalein. As soon as the free acid is neutralized any further addition of sodium carbonate produces a pink color. In using distilled water where there are no complications due to the equilibrium of the dissolved carbonates and the free carbon dioxide in solution, the method should give reliable results.

We have applied our method to the determination of free carbon dioxide in distilled water and have found it to be in excellent agreement with the Seyler method. The same general procedure was carried out in determining the carbon dioxide, as was used for the determination of dissolved oxygen. The same formula as was used in calculating the amount of dissolved oxygen is applicable and was made use of in the following determinations. (See Table IV.)

TABLE IV.-To ShOW AGREEMENT with THE SEYLER METHOD.

$\begin{array}{lllccc}\begin{array}{c}\text { Amt. of gas } \\ \text { removed. }\end{array} & \begin{array}{c}\text { Equit. } \\ \text { temp. }\end{array} & \begin{array}{c}\text { Anal. } \\ \text { temp. }\end{array} & \begin{array}{c}\text { Bar } \\ \text { pres. }\end{array} & \text { New meth. } \\ 0.45 \mathrm{cc} . & 20 . \mathrm{I}^{\circ} & 20.0^{\circ} & 749.49 & \mathrm{~S} \text { Seyler meth. } \\ 0.46 & 19.6 & 20.0 & 749.49 & \mathrm{I} .77 & \mathrm{I} .8 \mathrm{I} \\ 0.46 & 21.4 & 20.5 & 749.49 & \mathrm{I} .7 \mathrm{O} & \mathrm{I} .8 \mathrm{I} \\ 0.14 & 25.0 & 20.0 & 753.60 & 0.5 \mathrm{I} & 0.5 \mathrm{I} \\ 0.14 & 24.7 & 20.0 & 753.60 & 0.5 \mathrm{I} & 0.5 \mathrm{I} \\ 0.14 & 24.8 & 20.0 & 753.60 & 0.50 & 0.5 \mathrm{I}\end{array}$

TABLE V.-To Show the Reproductrility of OUR Method.

$\begin{array}{llllll}0.53 & 21.7 & 23.3 & 764.80 & 2.01 & 2.00 \\ 0.52 & 21.0 & 23.1 & 764.80 & 1.99 & 2.00 \\ 0.52 & 21.4 & 23.5 & 765.20 & 1.98 & 2.00 \\ 0.52 & 21.2 & 23.0 & 764.80 & 2.02 & 2.00 \\ 0.53 & 22.2 & 23.3 & 760.10 & 1.98 & 2.00\end{array}$

\section{Nitrogen.}

From a survey of the literature it was found that a little work has been done on the determination of dissolved nitrogen in water. There seems to be no valid reason why a knowledge of the amount of the dissolved nitrogen would not find an important place in the analysis of waters and effuents were it possible to determine it readily.

We were particularly interested when designing our method to make

i N. Rep. Pharm., 19, 1 .

${ }^{2}$ Chem. News, 70, 104.

3 This JoUrNaI, 23, 4 O5 (IgOI). 
it general in application and thereby the determination of dissolved nitrogen of no less importance than that of dissolved oxygen and carbon dioxide now so widely used for the studies of polution in harbors and river waters in this country and in England.

The only attempt to determine the dissolved nitrogen in water has been along the lines of completely removing the dissolved nitrogen by boiling out the gas under reduced pressure. One has, however, but to turn to the work of Roscoe and Lunt ${ }^{1}$ or Birge and Juday ${ }^{2}$ on the determination of dissolved nitrogen to learn of the experimental difficulties encountered by this general method.

By the application of our method we have determined the dissolved nitrogen in water very readily. We have checked the results obtained experimentally by the calculation of the theoretical amount thoroughly aerated water can hold at the particular temperature and pressure. We found that the calculated value was in excellent agreement with the amount determined by experiment.

In the following tables are given the results obtained from a series of duplicate determinations using distilled water:

TABLE VI.

$\begin{array}{ccccc}\begin{array}{c}\text { Amt. of gas } \\ \text { removed. }\end{array} & \begin{array}{c}\text { Equij. } \\ \text { temp. }\end{array} & \begin{array}{c}\text { Anal. } \\ \text { temp. }\end{array} & \begin{array}{c}\text { Bar. } \\ \text { pres. }\end{array} & \begin{array}{c}\text { New method. } \\ \text { C. nitrogen } \\ \text { per liter. }\end{array} \\ 8.06 \mathrm{cc} . & 22.2^{\circ} & 24.0^{\circ} & 761.80 \mathrm{~mm} . & 15.28 \mathrm{cc} . \\ 8.06 & 21.6 & 23.5 & 761.80 & 15.3 \mathrm{I} \\ 8.06 & 22.2 & 23.3 & 760.10 & 15.33 \\ 8.08 & 21.7 & 23.3 & 764.80 & 15.46 \\ 8.08 & 21.0 & 23.1 & 764.80 & 15.45 \\ 8.07 & 21.2 & 23.0 & 764.80 & 15.44 \\ & & \text { TABLE VII. } & & \\ 6.52 & 23.0 & 23.5 & 728.40 & 12.18 \\ 6.54 & 23.4 & 24.0 & 726.80 & 12.17 \\ 6.53 & 23.4 & 24.2 & 726.83 & 12.16 \\ 6.52 & 22.6 & 23.5 & 727.75 & 12.17 \\ 6.51 & 22.9 & 23.8 & 727.36 & 12.14 \\ 6.50 & 23.0 & 23.0 & 729.29 & 12.15\end{array}$

Another Apparatus for the Determination of Dissolved Oxygen.

The principle of the method already described by us for the determination of dissolved gases in water depends upon the establishment of an equilibrium between the gas in the gas phase and that in solution. This was accomplished by bringing the water into a vacuum and shaking until equilibrium was established. We could, however, just as well have used an indifferent ges as a vacuum and by shaking it with the water under examination established an equilibrium between the two phases. When the equilibrium is established the gas phase can be removed and

1 J. Chem. Soc., 55, 552 (1889).

${ }^{2}$ Wisc. Survey Bull., 23 (1911). 
analyzed for the gas under consideration. The total amount of the gas dissolved in the water may be calculated by the aid of the formula previously mentioned.

In the determination of dissolved oxygen by this scheme hydrogen was first used as the indifferent gas in which the equilibrium was established. A glass bulb of a liter capacity was filled with the water under consideration. Hydrogen was then allowed to displace half of the water, after which the equilibrium was established by vigorous shaking of the bulb containing the water and the indifferent gas. The gas phase was then removed and measured in a specially designed buret. A platinum wire within the buret was then brought to a bright glow by an electric current, whereby the hydrogen and oxygen in the mixture of gases were caused to combine to form water, i. e., $2 \mathrm{H}_{2}+\mathrm{O}_{2} \rightarrow 2 \mathrm{H}_{2} \mathrm{O}$, which condensed upon the walls of the buret. We have here the advantage of a contraction of three volumes for each volume of oxygen, but we have here also the disadvantage due to the fact that the volume of the oxygen was only I to $2 \%$ of the total gas volume. If the total volume was Ioo cc., then the volume measurements should be reliable to $0.01 \mathrm{cc}$ or less, in order to secure an accuracy of $0.1 \%$ of the oxygen. This means a more accurate control of the temperature and pressure before and after burning out the oxygen than can conveniently be secured in routine work, therefore, we sought other ways of determining this oxygen.

Oxygen may be readily removed from a mixture with an indifferent gas by copper, so we brought our sample of water into a nitrogen atmosphere and established equilibrium. The gas phase was then passed through potassium hydroxide solution to remove carbon dioxide, then over glowing copper, which picks up the oxygen. The amount of oxygen uniting with the copper was then determined indirectly by reheating the copper glower in the presence of hydrogen; the amount of hydrogen necessary to effect complete reduction to metallic copper being measured, the amount of oxygen being one-half of this amount. By this means we were able to obtain measurements on a relatively small volume of hydrogen and consequently we could readily obtain the desired accuracy. If, however, $\mathrm{CH}_{4}$ or organic vapor of any kind gets into the gas phase they would be oxidized by the $\mathrm{CuO}$ and $\mathrm{O}_{2}$ and so too little $\mathrm{O}_{2}$ found. This difficulty could be overcome, as the $\mathrm{N}_{2}$ which passed would contain the $\mathrm{CO}_{2}$ formed from the combustion of $\mathrm{CH}_{4}$ and it could be determined, but in our work we have had no evidence of the presence of oxidizable gases or vapors in the gas phase. The apparatus we used to carry out this idea is shown in Fig. 2.

The apparatus consists of a glower, A, made by winding nichrome resistance ribbon spirally upon a quartz tube $28 \mathrm{~cm}$. in length and $3 \mathrm{~mm}$. internal diameter, $5 \mathrm{~mm}$. external diameter. Fourteen centimeters of the tube 
were heated by the resistance ribbon, within which region strips of pure copper foil one-thousandth of an inch thick were placed in such a manner as to permit of maximum surface exposed. The use of copper of extreme thinness was found necessary, owing to the difficulty encountered when using thick copper of a slow rate of reduction of the copper oxides by hydrogen, the final value being obtained only after several heatings. This effect was probably due to the formation of curpous oxide, which penetrated beneath the surface of the copper. By the use of the very thin copper foil this slow rate of reduction was obviated and one heating of the glower to $5.50-600^{\circ} \mathrm{C}$. from 3 to 4 minutes was sufficient. The ends of the quartz

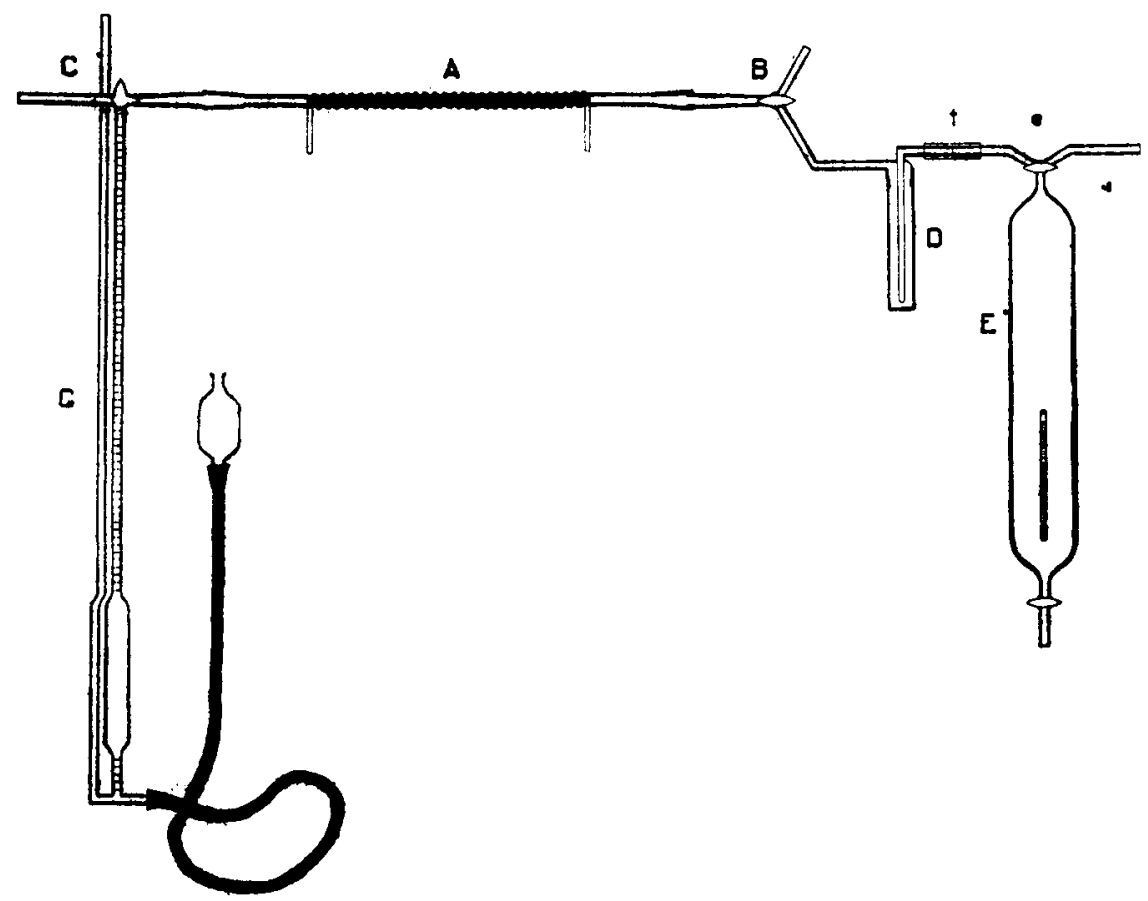

Fig. 2.

tubes were flared to meet a glass complement which was ground to fit; to make these joints perfectly secure a thin coating of hard Khotinsky cement was applied and the two parts fitted together while hot. At either end of the short glass capillaries joined to the quartz tube were sealed three-way stopcocks, viz., B and C, Fig. 2. One of the arms of the cock $\mathrm{B}$ was connected to a hydrogen generator by means of a glass tube in which was placed a small platinum glower. The glower served to free the hydrogen gas from any possible oxygen or organic matter that it might contain. The other arm of cock $B$ was joined to a wash bottle, D, of special design, through which the gases, as they came from bulb $\mathrm{E}$, were 
bubbled to remove carbon dioxide. To one of the arms of the 3-way cock $C$ at the other end of the quartz tube there was connected a calibrated gas buret, $G$, graduated to read to hundredths of cubic centimeters. To this buret was fitted a mercury leveling bulb and a manometer to aid in obtain accurate readings. The other arm of the cock $\mathrm{C}$ was connected to the air through a small wash bottle.

The water to be examined was drawn directly into a glass bulb, E, of a liter capacity. It was fitted with a 3-way cock on either end and carried a short range thermometer within graduated to read to tenths of degrees. This bulb was arranged on a carriage so that it could be easily removed in order to shake the contents. After the bulb $\mathrm{E}$ was filled with the water it was connected to a source of oxygen-free nitrogen by one of the arms of the upper cock; water was then drawn from the bulb by opening the lower cock until half of the water in the bulb was displaced by the nitrogen gas. The cocks were then closed, the bulb $\mathrm{E}$ removed from the carriage and shaken vigorously for five minutes, after which time the gas in the gas phase and the gas in solution were in equilibrium. The bulb was then replaced upon the support and connected to the glower by means of a ground-glass joint. The gas above the water was then displaced without changing the pressure by permitting water to enter the bulb $E$ through the lower cock, from a reservoir kept at a temperature corresponding to the temperature within the bulb. The gas displaced from the bulb was bubbled through caustic potash solution in the washer $B$ and thence into the quartz glower where the oxygen was picked up by the glowing copper. When the gas had all been displaced from bulb $\mathrm{E}$ the cock $e$ was turned to permit the system to be swept out by nitrogen in order to carry through the glower any oxygen in the wash bottle or connections. The glower was then allowed to cool in a nitrogen atmosphere. When it had reached room temperature hydrogen gas was passed through the glower and after the system had been thoroughly swept out by the hydrogen, the cock $\mathrm{C}$. was turned to permit the gas buret $\mathrm{G}$ to become filled, then cock B was closed and the current again turned onto the glower. The copper which was in part oxidized in the passage of the oxygen laden nitrogen from the bulb $E$ was now reduced by the hydrogen entering the glower from the buret as fast as it was used up in reduction. The water formed from the union of the hydrogen and oxygen condensed upon the cold walls of the glower. After complete reduction the glower was allowed to cool to room temperature when the difference in the readings of the mercury in the buret gave the amount of hydrogen which had been used to effect reduction, account being taken of the volume occupied by the condensed water and change in volume of $\mathrm{CuO}$ going to $\mathrm{Cu}$. The amount of hydrogen in the buret before reduction was usually $8 \mathrm{cc}$., after reduction 0.5 to I.O cc: remained in buret. The 
amount of oxygen actually removed from the sample of water was equal to one-half of the hydrogen volume used up in the reduction as the combining volume; ratios of oxygen to hydrogen is as $I: 2$. From the amount of oxygen thus determined a knowledge of the actual amount of dissolved oxygen in the sample of water was obtained by use of the formula given in the first part of this paper.

It was evident when making a run that all of the oxygen was being picked up, as a considerable portion of the glowing copper opposite the end at which the gas mixture entered remained bright, showing no trace of oxidation.

In the following table are given the results obtained by the use of this method in determining the amount of dissolved oxygen in distilled water. Both the Winkler ${ }^{1}$ and our first method for determining oxygen were run as checks on the above method.

\begin{tabular}{|c|c|c|c|c|c|}
\hline $\begin{array}{l}\text { Amt. of oxygen } \\
\text { removed. }\end{array}$ & $\begin{array}{l}\text { Equil. } \\
\text { temp. }\end{array}$ & $\begin{array}{l}\text { Room } \\
\text { temp. }\end{array}$ & $\begin{array}{c}\text { Bar. } \\
\text { pressure. }\end{array}$ & \multicolumn{2}{|c|}{$\begin{array}{c}\text { New meth. Winkler meth. } \\
\text { Cc. per liter. }\end{array}$} \\
\hline $3.22 \mathrm{cc}$. & 21.0 & 20.8 & $755 . \infty 0$ & 6.42 & 6.40 \\
\hline 3.2 .5 & 20.5 & 21.0 & $75.5 \cdot \mathrm{m}$ & $6.4^{8}$ & 6.40 \\
\hline 3.26 & 21.8 & 20.5 & 755.00 & 6.49 & 6.46 \\
\hline .3 .22 & 21.8 & 20.5 & 755.00 & 6.43 & 6.45 \\
\hline 3.25 & 21.8 & 20.5 & $75.5 . \infty$ & 6.47 & 6.44 \\
\hline 3.20 & 21.5 & 20.0 & 755.02 & 6.39 & 6.40 \\
\hline 3.22 & 21.7 & 20.1 & 755.02 & 6.42 & 6.40 \\
\hline 3.20 & 216 & 20.0 & $7.5 .5 \cdot 02$ & 6.40 & 6.40 \\
\hline 3.22 & 21.5 & 20.2 & 755.02 & 6.42 & 6.40 \\
\hline .3 .24 & 21.7 & 20,0 & $7.54 \cdot(x)$ & 6.47 & 6.40 \\
\hline 3.20 & $21 .+4$ & 20.1 & 754.00 & 6.39 & 6.40 \\
\hline
\end{tabular}

Laboratory of Physical Chgmistry.

Princeton, N. J.

[CONTRibUtion From the JohN Harrison Laboratory of Chemistry.]

\section{OBSERVATIONS UPON THE QUANTITATIVE DETERMMNATION OF FLUORINE.}

BY WILLIAM H. ADOLPH.

Received September 4, 1915.

Berzelius ${ }^{2}$ originally precipitated and weighed flworine as calcium fluoride. Rose ${ }^{3}$ suggested the precipitation of the fluoride in the presence of carbonate to facilitate filtering. The ignition of this combined precipitate and its subsequent extraction with acetic acid left calcium fluoride in a granular form which was easily filtered. The extraction has usually been made with I.5 $N$ acetic acid. To test the solubility of calcium

: Ber, 2 I, 2843 (1888).

S Schweigg. Jour., 16, 426 (1816).

${ }^{3}$ Leib. Ann., 72, 343 (1849). 